\section{Immunohistochemical expression of heparanase isoforms and syndecan-1 proteins in colorectal adenomas}

\author{
J. Waisberg, T.R. Theodoro, L.L. Matos, \\ F. Brasil, R.L. Serrano, G.T. Saba, \\ M.A.S. Pinhal \\ ABC Medical School, Santo André, SP, \\ Brazil
}

\section{Abstract \\ The proteoglycan syndecan-1 and the} endoglucuronidases heparanase- 1 and heparanase- 2 are involved in molecular pathways that deregulate cell adhesion during carcinogenesis. Few studies have examined the expression of syndecan-1, heparanase- 1 and mainly heparanase-2 proteins in non-neoplastic and neoplastic human colorectal adenoma tissues. The aim of this study was to analyze the correlation among the heparanase isoforms and the syndecan-1 proteins through immunohistochemical expression in the tissue of colorectal adenomas. Primary antihuman polyclonal anti-HPSE and anti-HPSE2 antibodies and primary anti-human monoclonal anti-SDC1 antibody were used in the immunohistochemical study. The expressions of heparanase-1 and heparanase-2 proteins were determined in tissue samples from 65 colorectal adenomas; the expression of syndecan-1 protein was obtained from 39 (60\%) patients. The histological type of adenoma was tubular in $44(67.7 \%)$ patients and tubular-villous in 21 (32.3\%); there were no villous adenomas. The polyps were $<1.0 \mathrm{~cm}$ in size in 54 (83.1\%) patients and $\geq 1.0 \mathrm{~cm}$ in 11 (16.9\%). The images were quantified by digital counter with a computer program for this purpose. The expression index represented the relationship between the intensity expression and the percentage of positively stained cells. The results showed that the average of heparanase-1, heparanase-2 and syndecan-1 expression index was 73.29 o.u./. $\mathrm{m}^{2}, 93.34$ o.u. $/ \mathrm{\mu m}^{2}$, and 55.29 0.u. $/ \mathrm{\mu m}^{2}$, respectively. The correlation between the heparanase-1 and syndecan-1 expression index was positive $(\mathrm{R}=0.034)$ and significant $(\mathrm{P}=0.035)$. There was a negative $(\mathrm{R}=-0.384)$ and significant $(\mathrm{P}=0.016)$ correlation between the expression index of heparanase-1 and heparanase-2. A negative $(\mathrm{R}=-0.421)$ and significant $(\mathrm{P}=0.008)$ correlation between the expression index of heparanase-2 and syndecan-1 was found. We concluded that in colorectal adenomas, the heparanase-1 does not participate in syndecan-1 degradation; the heparanase-2 does not stimulate syndecan-1 degradation by the action of heparanase-1, and the heparanase-2 may be involved in the modulation of the heparanase-1 activity.

\section{Introduction}

Patients with colorectal adenomas exhibit a threefold-increased risk of developing colorectal cancer, a risk that is even greater among patients who are older than 60 years and/or those presenting with multiple colorectal adenomas. ${ }^{1}$

Although every adenoma has the capacity for malignant evolution, most adenomas can stabilize their growth, and few develop invasive cancer. The potential for malignant evolution is in fact correlated with adenoma size, growth pattern, and grade of dysplasia. ${ }^{2,3}$

The adenoma-carcinoma sequence of the large bowel is a worthy model of tumor progression according to which the accumulation of genetic alterations in the neoplastic clone leads to the rise of tumoral subpopulations with selection and clonal expansion of those variants with phenotypic and growth advantages. ${ }^{4,5}$ Histologically, adenomas with low- and high-grade dysplasia are intermediate steps, and carcinoma infiltrating the intestinal wall at various levels is the end point of the sequence. ${ }^{6,7}$ Genes upregulated in adenoma relative to normal tissue, which maintained increased expression in colorectal carcinoma and adenoma, would encode proteins suitable as putative targets for immunoprevention. ${ }^{4,5,8}$ The identification of early and easily detectable tumor markers that might contribute to the knowledge of colorectal carcinogenesis and the biological mechanisms required for preinvasive adenoma to progress to carcinoma, are highly relevant subjects. ${ }^{4,5}$ The process of proliferation and tumor invasion depends, among other factors, on changes in the extracellular matrix (ECM) represented by the loss of cell-cell interaction, ECM degradation by tumor cells through activation of enzymes associated with neoplastic invasion that disorganize and fragment the stromal elements and their own ECM, and the synthesis of ECM components by metastatic tumor cells. ${ }^{9-11}$

Heparan sulfate (HS) proteoglycans are important constituents of the cell membrane and they act as co-receptors for cellular signaling. ${ }^{12,13}$ Cell surface HS proteoglycans are regulators of the migratory, mitogenic, secretory and inflammatory activities of the cell. The HS proteoglycans bind to the soluble heparinbinding growth factors and present them to their respective signaling receptors. ${ }^{10,14}$ The
Correspondence: Dr. Jaques Waisberg, ABC Medical School, Av. Príncipe de Gales 821, 09060650 Santo André, SP, Brazil.

Tel. +55.11.98256-0018 - Fax: +55.11.4436-8739

E-mail: jaqueswaisberg@uol.com.br

Key words: Syndecan-1; colorectal neoplasms immunohistochemistry; heparan sulfate proteoglycans; glucuronidase; carcinogenesis.

Contributions: MASP, protocol and data analysis, preparation, interpretation of analysis results, manuscript revision; TRT, technical analysis, data analysis; FB, technical analysis, partial manuscript revision; RLS, technical analysis; LLM, interpretation of analysis results, statistical study; GTS, manuscript language and stylistics, partial manuscript revision; JW, final data analysis, final manuscript revision.

Conflict of interest: the authors declare no conflict of interest.

Received for publication: 23 November 2015 Accepted for publication: 8 February 2016.

This work is licensed under a Creative Commons Attribution-NonCommercial 4.0 International License (CC BY-NC 4.0).

(C) Copyright J. Waisberg et al., 2016

Licensee PAGEPress, Italy

European Journal of Histochemistry 2016; 60:2590 doi:10.4081/ejh.2016.2590

development of a tumor in the large bowel is often associated with loss of normal epithelial adhesion and altered patterns of expression of cell adhesion molecules. ${ }^{15}$

Syndecan-1 (SDC1) is a member of the syndecan family that comprises a transmembrane HS proteoglycan. Syndecan-1 has important biological functions at the surface of epithelial cells, ${ }^{11,14}$ and is essential for cell-cell and cellmatrix interactions. SDC1 is encoded by the gene $S D C 1$ located in chromosome 2p24.1. This molecule acts as an extracellular matrix receptor and is involved in many cellular functions, including cell binding, cell signaling, and cytoskeletal organization through cell-cell adhesion and cell-matrix adhesion. ${ }^{16}$ In adult human tissues, SDC1 is predominantly expressed in epithelial cells and plasma cells. Syndecan-1 is involved in molecular pathways that are deregulated during carcinogenesis and are related to cell proliferation, tumor adhesion, apoptosis, angiogenesis, tumor invasion, and metastasis. ${ }^{17,18}$

Heparanase is an endoglucuronidase that cleaves HS chains of proteoglycans at specific intrachain sites in the extracellular matrix and play an important role in the extravasation of blood-borne tumor cells and inflammatory leukocytes. ${ }^{19,20}$ Upon degradation, heparanase releases growth factors and cytokines that 
stimulate cell proliferation and chemotaxis. ${ }^{21,22}$ Heparanase activity plays a decisive role in cell dissemination associated with cancer metastasis, and in many malignancies, the high expression and activity of heparanase correlate with an aggressive tumor phenotype. .1,23-24 $^{2}$

There are two isoforms of heparanase. The human gene heparanase (HPSE) encoding heparanase-1 isoform (HPSE) maps to chromosome $4 q 21.23 .^{23}$ HPSE dismantles the subendothelial basal membrane and facilitates the blood-borne metastasis of tumor cells by the cleavage of the heparan sulfate chains from proteoglycans. Furthermore, HPSE induces angiogenesis and promotes the vascularization of tumors..$^{10,21}$ HPSE is involved in the degradation of HS in both the cell-surface, and ECM in non-neoplastic and neoplastic tissues. ${ }^{12,20}$ The gene HPSE2 encoding heparanase-2 isoform (HPSE2) maps to human chromosome 10q24.2. ${ }^{25}$ The HPSE2 is an intracellular protein associated with the cell membrane and can be found in the brain, small intestine, prostate, mammary gland, testis, and uterus. ${ }^{26-28}$ The HPSE2 isoform has no enzymatic activity and appears to play a role in regulating HPSE activity. ${ }^{12,20}$ HPSE2 is overexpressed in colorectal carcinoma tissues compared with non-neoplastic tissues, and this phenomenon could be possibly related to SDC1 shedding even though HPSE2 does not present enzymatic activity. ${ }^{20,25}$ Few studies have examined the expression of SDC1, HPSE and mainly HPSE2 in non-neoplastic and neoplastic colorectal adenoma tissues. . $^{7,13,15,29}$

The aim of this study was to study the immunohistochemical expression of SDC1, HPSE and HPSE2 proteins in colorectal adenomas tissue samples.

\section{Materials and Methods}

All procedures performed in studies involving human participants were in accordance with the ethical standards of the institutional and/or national research committee and with the 1964 Helsinki declaration and its later amendments or comparable ethical standards. Informed consent was obtained from all individual participants included in the study.

This work is an observational, longitudinal, and retrospective study on patients who underwent colorectal adenoma resection between 2012 and 2013.

The expressions of HPSE and HPSE2 were determined in tissue samples from 65 colorectal adenomas obtained from 65 patients. Thirty-eight (58.5\%) were men and 27 (41.5\%) were women. The average age was $62.1 \pm 11.1$ years (29-88 years). The polyps were located in the colon in $59(90.8 \%)$ cases and in the rec- tum in $6(9.2 \%)$. The average size of the polyps was $0.7 \pm 0.3 \mathrm{~cm}(0.3$ to $1.8 \mathrm{~cm})$. The polyps were $<1.0 \mathrm{~cm}$ in size in $54(83.1 \%)$ patients and $\geq 1.0 \mathrm{~cm}$ in 11 (16.9\%). The histological type of adenoma was tubular in 44 (67.7\%) patients and tubular-villous in 21 (32.3\%). There were no villous adenomas. The degree of atypia observed was mild in 43 (66.1\%), moderate in 17 (26.2\%) and severe in 5 (7.7\%) adenomas. In 39 (60\%) patients, the level of
SDC1 protein was also obtained in colorectal adenoma tissue.

Samples containing specimens of colorectal adenomas obtained by endoscopic polypectomy were fixed in $10 \%$ buffered formalin, routinely processed, and paraffin embedded. The paraffin blocks were cut 3-mm thick, and slides were prepared for immunohistochemical study according to protocol used in our laboratory and previously described. ${ }^{30}$ Briefly, endoge-

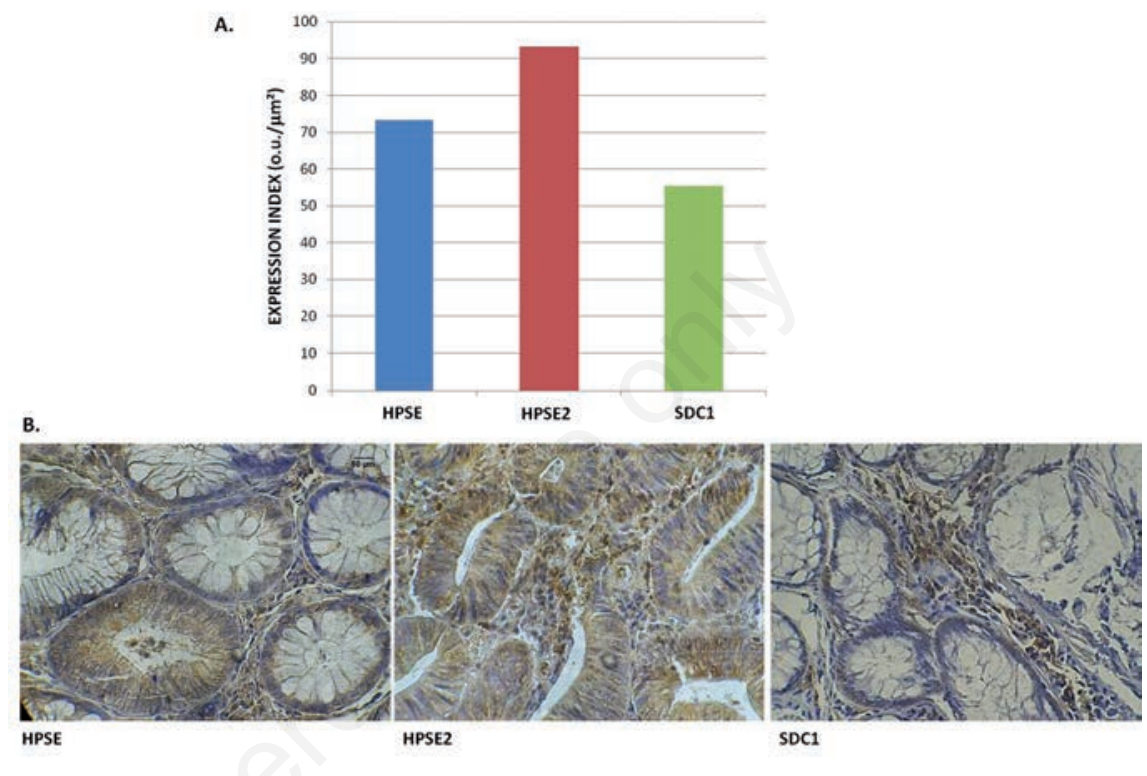

Figure 1. A) Expression index (EI) of anti-heparanase-1 (HPSE), anti-heparanase-2 (HPSE2), and anti-syndecan-1 (SDC1) antibodies in colorectal adenomas; the values obtained represent the mean of assays performed in triplicate and are expressed in o.u./ $\mu^{2}{ }^{2}$. B) Photomicrography of the immunoreactivity of the antibodies cited (Immunohistochemistry, 200X).

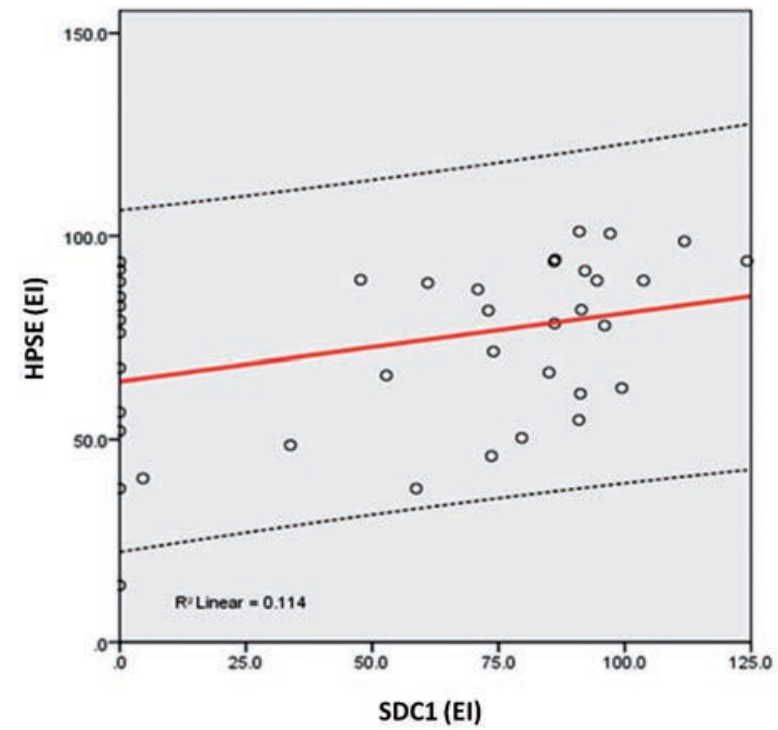

Figure 2. Relationship between the expression index of heparanase-1 (HPSE) and syndecan-1 (SDC1). The values were obtained as described in Methods and represent the expression assays performed in triplicate index (values expressed in $0 . u . / \mu^{2}{ }^{2}$. 
nous peroxidase activity was blocked by incubating the sections in a methanol bath containing 3\% hydrogen peroxide for $20 \mathrm{~min}$, followed by a washing in distilled water. The sections were initially submitted to heat-induced epitope retrieval using citrate buffer $(\mathrm{pH} 9.0)$ in an uncovered pressure cooker (Eterna ${ }^{\circledR}$; Nigro, Araraquara, SP, Brazil). Blocking of endogenous peroxidase was obtained with $3 \%$ $\mathrm{H}_{2} \mathrm{O}_{2}$ (10 vol.), with 3 washes of 10 -min each. The slides were again washed in distilled running water and then in phosphate-buffered saline (10 $\mathrm{mM} ; \mathrm{pH} 7.4)$ for $5 \mathrm{~min}$. Subsequently, the primary antibody was applied and the slides were incubated overnight at $8^{\circ} \mathrm{C}$.

Primary anti-human polyclonal anti-HPSE (HPA1 H-80) and anti-HPSE2 (HPA2 C-17) antibodies purified in goat (Santa Cruz Biotechnology®, Santa Cruz, CA, USA) were used at a dilution of 1:100. Primary antihuman monoclonal anti-SDC1 (anti-CD138) purified in mouse (AbD Serotec ${ }^{\circledR}$, Bio-Rad Company Co., Oxford, UK) was used at a dilution of 1:50. Immunohistochemical staining was performed using the biotinylated secondary antibody obtained from the avidin-biotinperoxidase complex (LSAB + System-HRP, Dako North America, Inc., Carpinteria, CA, USA). As a positive control, a histological section of a bronchopulmonary carcinoid tumor was used. A similar histological section without the primary antibody reaction was used as a negative control.

Slide analysis was conducted with an optical microscope (Eclipse TS100 Light, Nikon Corporation $®$, Tokyo, Japan). For each slide, an average of 15 pictures of $640 \times 480$ pixels were obtained with $400 \mathrm{x}$ magnification by a camera (model 4300, Nikon Coolpix Digital, Nikon Corporation $\AA$, Tokyo, Japan). The images were quantified by digital counter with a computer program for this purpose according to the protocol used in our laboratory. ${ }^{30}$ The staining intensity was quantified by corresponding to the intensity expression (ItE) in HPSE, HPSE2, and SDC1; the percentage of positively stained cells (PI); and the expression index (EI) comprising the relationship between PI and ItE. The digital expression indices of HPSE, HPSE2 and SDC1 were expressed in optic units per square micrometer (0.u./ $\left.\mathrm{\mu m}^{2}\right)$.

The images were quantified by digital software counter (ImageLab 2000 ${ }^{\mathrm{TM}}$, Softium Informática, São Paulo, SP, Brazil), adjusted to a micrometer scale. This program was used to quantify the intensity of staining corresponding to the expression of the analyzed marker index (ITE), the percentage of positively stained cells (PI) and expression index (IE) comprising the relationship between the PI and the ITE. The methodology used to deter- mine the digital expression index of each marker of the present study was previously determined by our group. ${ }^{30}$ Briefly, for each adenoma, about 500 of colonic mucosa cells observed in the photographs were counted by the digital counter and classified by the observer as positive (marked) or negative (unmarked) cells. Subsequently, using the same images, the calibration program was conducted to micrometer range, then the size of the area configuration that had the measured density. Then, it was performed to determine the 12 areas of stained cells (three quadrant of the image) and an automatic calculation of the optical density of areas was obtained. Finally, the obtained RGB (Red, Green, and Blue) average per area was expressed in $0 . u . / \mathrm{mm}^{2}$. The same steps were followed to calculate the optical density of the white background (no tissue area) of each his-

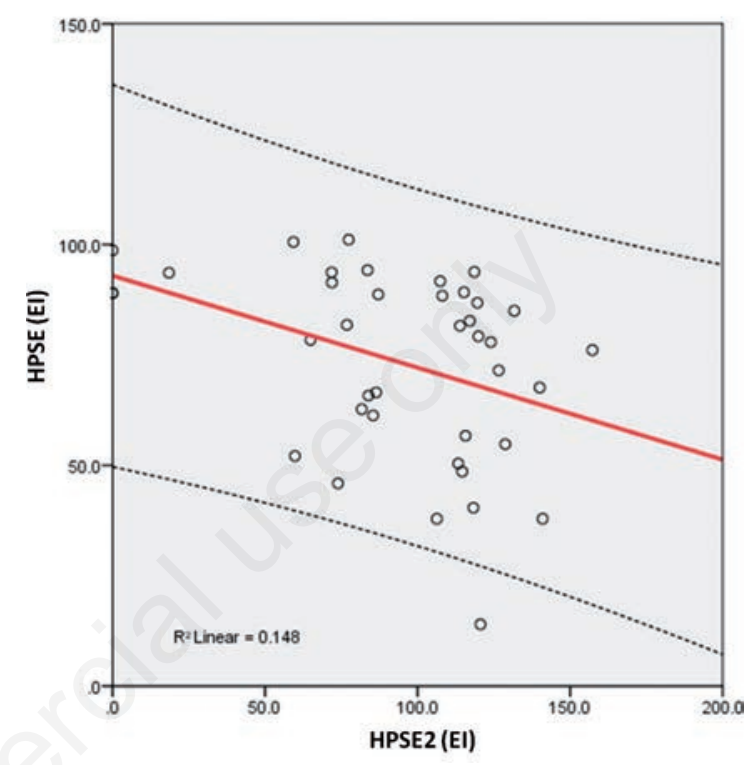

Figure 3. Relationship between the expression index of heparanase-1 (HPSE) and heparanase-2 (HPSE2). The values were obtained as described in Methods and represent the expression assays performed in triplicate index (values expressed in $0 . u . / \mu \mathrm{m}^{2}$ ).

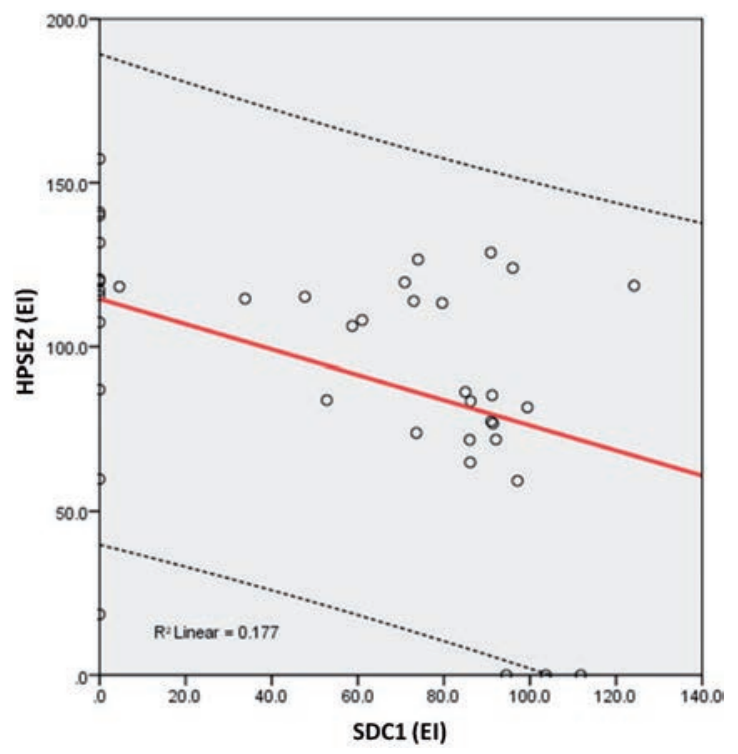

Figure 4. Relationship between the expression index of heparanase-2 (HPSE2) and syndecan-1 (SDC1). The values were obtained as described in Methods and represent the expression assays performed in triplicate index (values expressed in $0.0 . / \mu^{2}$ ). 
tological slide (background control reaction).

The Kolmogorov-Smirnov test was used to verify the data's normal distribution. Statistical associations between the protein levels of HPSE, HPSE2, and SDC1 were determined using Mann-Whitney's test, Chi-square $\left({ }^{2}\right)$, and Fisher's exact test for frequency and proportion comparisons. To measure the association between protein levels of HPSE, HPSE2, and SDC1 as ordinal variables, the Spearman correlation coefficient (R) was used. The statistical significance level was set at $5 \%(\mathrm{P}<0.05)$, and the data were analyzed using the SPSS ${ }^{\circledR}$ software (Statistical Package for Social Sciences; SPSS, Chicago, IL, USA), version 17.0.

\section{Results}

In 65 cases of colonic adenomas labeled we utilized anti- HPSE and anti- HPSE2 anti-antibodies; in 39 of these cases it was also used anti- SDC1 antibody. The average EI of HPSE, HPSE2 and SDC1 was 73.29 o.u./$/ \mathrm{m}^{2}, 93.34$ o.u./$/ \mathrm{\mu m}^{2}$, and 55.29 o.u./$/ \mathrm{m}^{2}$, respectively (Figure 1).

Immunohistochemical evaluation demonstrated that HPSE is mainly distributed in epithelial cells of the dysplastic glands and in the extracellular matrix, while HPSE2 presented a higher expression in the adenoma tissue compared to the HPSE. Furthermore, SDC1, a major heparan sulfate proteoglycan that is mainly localized at the cell surface, can be detected in the lamina propria, suggesting the action of HPSE and shedding of this proteoglycan. The correlation between the immunohistochemical level of HPSE and SDC1 was positive $(\mathrm{R}=0.034)$ and significant $(\mathrm{P}=0.035)$ (Figure 2). There was a negative $(\mathrm{R}=-0.384)$ and significant $(\mathrm{P}=0.016)$ correlation between the EI of HPSE and HPSE2 (Figure 3). A negative $(R=-0.421)$ and significant $(P=0.008)$ correlation between the EI of HPSE2 and SDC1 was found (Figure 4).

As a corollary of the results obtained in this study, it was observed also that no significant differences were observed between the expressions of HPSE, HSPE2 and SDC1, and the clinicopathological parameters (age and gender of patients, and degree of atypia, histological type, size and location of adenoma) of the colorectal adenomas.

\section{Discussion}

The heparanase acts as a master regulator of the aggressive tumor phenotype in part by enhancing expression of proteins known to drive tumor progression like VEGF, MMP-9 and hepatocyte growth factor (HGF). ${ }^{9,13,18,31,32}$ HPSE is the unique and specific functional endoglycosidase capable of cleaving HS chains in mammalian cells. ${ }^{10,16}$ Oligosaccharides generated by HPSE enhance angiogenesis and inflammation process. ${ }^{13,20,33}$ SDC1 expression by tumor cells or the lack thereof has been associated with poor prognosis in several types of cancer, including colorectal cancer. ${ }^{15}$ Data have been reported showing that HPSE regulates SDC1 and promotes its shedding from the cell surface. ${ }^{14,34}$ Therefore, degradation of cell surface heparan sulfate chains of the SDC1 by HPSE increased the levels of SDC1 secretion to the lamina propria which seem to be important event in the carcinogenesis. Thus, it is evident why this enzyme may be an effective and attractive drug target. ${ }^{32,35}$ Several HPSE inhibitors have been developed, and some of them have undergone clinical trials showing efficacy against tumors. ${ }^{32}$

In the present study, the positive immunohistochemical expression of HPSE, HPSE2, and SDC1 proteins advocates that both isoforms of HPSE and SDC1 may possibly have some role in the neoplastic conversion of the colorectal adenoma. This explanation can also be suggested by the finding of increased immunohistochemical expression of the isoform HPSE2 and SDC1 in colorectal cancers as compared to non-neoplastic colorectal tissue. ${ }^{12}$ The results of our study indicated that in colorectal adenomas, HPSE immunohistochemical expression is increased, which was also observed in colorectal carcinoma. 15,29

There was a decrease in the SDC1 immunohistochemical expression directly proportional to the increase of HPSE, suggesting that HPSE enzyme is active in colorectal adenomas. Moreover, the present data corroborate with the literature results, which demonstrated that in the malignant tumors, including colorectal carcinoma, authors observed that increasing HPSE was directly related to the decrease of SDC1 immunohistochemical expression. ${ }^{12,34}$ Friedmann et al. ${ }^{29}$ related that the HPSE gene and protein were expressed already at the stage of colonic adenoma, but were practically not detected in the adjacent non-neoplastic colon epithelium. Gradually increasing expression of HPSE gene was evident as the cells progressed from severe dysplasia through welldifferentiated to poorly differentiated colon carcinoma.

Our results suggest that HPSE2 isoform could modulate the HPSE activity. The negative correlation observed in colorectal adenomas between HPSE and HPSE2 immunohistochemical expressions may indicate that HPSE2 negatively modulates HPSE; i.e., increasing levels of HPSE2 are possibly involved with the decreased activity of HPSE, contributing to the increase in the SDC1 immunostaining in the colorectal tissue. It is also possible that in adenomas, the HPSE exists in the form of a proenzyme or in a less active state compared with what occurs in carcinomas. Thus, this process may explain the negative relationship found between the SDC1 and HPSE2 in colorectal adenomas.

The tumor cell expression of HPSE regulates both the level and the location of SDC1 within the tumor microenvironment by enhancing the shedding of this molecule from the tumor cell surface. ${ }^{16,36}$ This regulation requires the presence of active enzyme, because mutated forms of HPSE lacking HS-degrading activity failed to influence SDC1 immunohistochemical expression. Removal of HS from the cell surface using bacterial heparitinase dramatically accelerated SDC1 shedding, suggesting that the effects of HPSE on SDC1 expression by tumor cells may be due, at least in part, to enzymatic removal or reduction in the size of HS chains. ${ }^{34}$

In this study, the positive correlation between the HPSE isoform and the SDC1 in colorectal adenomas suggests that HPSE isoform is involved in the SDC1 degradation, resulting in a decreased expression of SDC1 from cellular compartment and increased secretion of this molecule to the plasma. The inverse correlation between HPSE and HPSE2 indicates that the HPSE2 isoform probably has a regulatory function in HPSE activity. Preclinical and clinical studies have demonstrated that therapies targeting the HPSE/SDC1 axis hold promise for blocking the aggressive behavior of cancer. ${ }^{16,35}$ SDC1 plays a crucial role in carcinogenesis and is an attractive target for anticancer treatment with HPSE inhibitors and immunotherapy with anti-SDC1 antibodies. ${ }^{17,36}$

In conclusion, the heparanase-1 isoform in colorectal adenomas participate in syndecan-1 degradation and shedding; isoform of heparanase-2 may be involved in the modulation of the heparanase-1 activity. Taken together, these findings indicate the possible role of heparanases in the early events in colorectal carcinogenesis.

\section{References}

1. Shteingauz A, Ilan N, Vlodavsky I. Processing of heparanase is mediated by syndecan-1 cytoplasmic domain and involves syntenin and $\alpha$-actinin. Cell Mol Life Sci 2014;71:4457-70.

2. Fairley KJ, Li J, Komar M, Steigerwalt N, Erlich P. Predicting the risk of recurrent adenoma and incident colorectal cancer based on findings of the baseline 
colonoscopy. Clin Transl Gastroenterol 2014;5:e64.

3. Cao Y, Rosner BA, Ma J, Tamimi RM, Chan AT, Fuchs CS, et al. Assessing individual risk for high-risk colorectal adenoma at first-time screening colonoscopy. Int $\mathrm{J}$ Cancer 2015;137:1719-28.

4. Rosenberg DW, Giardina C, Tanaka T. Mouse models for the study of colon carcinogenesis. Carcinogenesis 2009;30:183-96.

5. Goel A, Boland CR. Recent insights into the pathogenesis of colorectal cancer. Curr Opin Gastroenterol 2010;26:47-52.

6. Casorzo L, Dell'Aglio C, Sarotto I, Risio M. Aurora kinase A gene copy number is associated with the malignant transformation of colorectal adenomas but not with the serrated neoplasia progression. Hum Pathol 2015;46:411-8.

7. Kaprio T, Satomaa T, Heiskanen A, Hokke $\mathrm{CH}$, Deelder AM, Mustonen H, et al. N-glycomic profiling as a tool to separate rectal adenomas from carcinomas. Mol Cell Proteomics 2015;14:277-88.

8. Nogueira RB, Pires AR, Soares TM, Rodrigues SR, Campos MA, Toloi GC, et al. Immunoexpression of the COX-2, p53, and caspase- 3 proteins in colorectal adenoma and non-neoplastic mucosa. Einstein (Sao Paulo) 2013;11:456-61.

9. Wang L, Ku XM, Li Y, Bian HJ, Zhang SH, Ye $\mathrm{H}$, et al. Regulation of matrix metalloproteinase production and tumor cell invasion by four monoclonal antibodies against different epitopes of HAb18G/CD147 extracellular domain. Hybridoma (Larchmt) 2006;25:60-7.

10. Levy-Adam F, Ilan N, Vlodavsky I. Tumorigenic and adhesive properties of heparanase. Semin Cancer Biol 2010;20:153-60.

11. Qing Q, Zhang S, Chen Y, Li R, Mao H, Chen $\mathrm{Q}$. High glucose-induced intestinal epithelial barrier damage is aggravated by syndecan-1 destruction and heparanase overexpression. J Cell Mol Med 2015;19:1366-74.

12. Kovalszky I, Hjerpe A, Dobra K. Nuclear translocation of heparan sulfate proteoglycans and their functional significance. Biochim Biophys Acta 2014;1840:2491-7.

13. Peretti T, Waisberg J, Mader AM, de Matos LL, da Costa RB, Conceição GM, et al. Heparanase-2, syndecan-1, and extracellular matrix remodeling in colorectal carcinoma. Eur J Gastroenterol Hepatol 2008;20:756-65.

14. Shteingauz A, Ilan N, Vlodavsky I. Processing of heparanase is mediated by syndecan-1 cytoplasmic domain and involves syntenin and $\alpha$-actinin. Cell Mol Life Sci 2014;71:4457-70.

15. Day RM, Hao X, Ilyas M, Daszak P, Talbot IC, Forbes A. Changes in the expression of syndecan-1 in the colorectal adenoma-carcinoma sequence. Virchows Arch 1999;434:121-5.

16. Ramani VC, Purushothaman A, Stewart MD, Thompson CA, Vlodavsky I, Au JL, et al. The heparanase/syndecan-1 axis in cancer: mechanisms and therapies. FEBS J 2013;280:2294-306.

17. Purushothaman A1, Uyama T, Kobayashi F, Yamada S, Sugahara K, Rapraeger AC, et al. Heparanase-enhanced shedding of syndecan-1 by myeloma cells promotes endothelial invasion and angiogenesis. Blood 2010;115:2449-57.

18. Chen L, Sanderson RD. Heparanase regulates levels of syndecan-1 in the nucleus. PLoS One 2009;4:e4947.

19. Vlodavsky I, Elkin M, Pappo O, Aingorn H, Atzmon R, Ishai-Michaeli $\mathrm{R}$, et al. Mammalian heparanase as mediator of tumor metastasis and angiogenesis. Isr Med Assoc J 2000;2 Suppl:37-45.

20. Sanderson RD, Yang Y, Suva LJ, Kelly T. Heparan sulfate proteoglycans and heparanase--partners in osteolytic tumor growth and metastasis: matrix biology. J Intern Soc Matrix Bio 2004;23:341-52.

21. Arvatz G, Shafat I, Levy-Adam F, Ilan N, Vlodavsky I. The heparanase system and tumor metastasis: is heparanase the seed and soil? Cancer Metastasis Rev 2011;30:253-68.

22. Rodrigues LM, Theodoro TR, Matos LL, Mader AM, Milani C, Pinhal MA. Heparanase isoform expression and extracellular matrix remodeling in intervertebral disc degenerative disease. Clinics (Sao Paulo) 2011;66:903-9.

23. Hulett MD, Freeman C, Hamdorf BJ, Baker RT, Harris MJ, Parish CR. Cloning of mammalian heparanase, an important enzyme in tumor invasion and metastasis. Nat Med 1999;5:803-9.

24. Reiland J, Sanderson RD, Waguespack M, Barker SA, Long R, Carson DD, et al. Heparanase degrades syndecan-1 and perlecan heparan sulfate: functional implications for tumor cell invasion. J Biol Chem 2004;279:8047-55.

25. Levy-Adam F, Feld S, Cohen-Kaplan V, Shteingauz A, Gross M, Arvatz G, et al. Heparanase 2 interacts with heparan sulfate with high affinity and inhibits heparanase activity. J Biol Chem 2010;285: 28010-9.

26. McKenzie E, Tyson K, Stamps A, Smith P,
Turner P, Barry R, et al. Cloning and expression profiling of HPSE2, a novel mammalian heparanase family member. Biochem Biophys Res Commun 2000;276:1170-7.

27. Navarro FP, Fares RP, Sanchez PE, Nadam $\mathrm{J}$, Georges B, Moulin C, et al. Brain heparanase expression is up-regulated during postnatal development and hypoxia-induced neovascularization in adult rats. J Neurochem 2008;105:34-45.

28. Suhovskih AV, Mostovich LA, Kunin IS, Boboev MM, Nepomnyashchikh GI, Aidagulova SV, et al. Proteoglycan expression in normal human prostate tissue and prostate cancer. ISRN Oncol 2013;2013: 680136.

29. Friedmann YI, Aingorn H, Aviv A, Peretz T, Pecker I, Pappo 0. Expression of heparanase in normal, dysplastic, and neoplastic human colonic mucosa and stroma. Am J Pathol 2000;157:1167-75.

30. Matos LL, Stabenow E, Tavares MR, Ferraz AR, Capelozzi VL, Pinhal MA. Immunohistochemistry quantification by a digital computer-assisted method compared to semiquantitative analysis. Clinics (Sao Paulo) 2006;61:417-24.

31. Purushothaman A, Hurst DR, Pisano C, Mizumoto S, Sugahara K, Sanderson RD. Heparanase-mediated loss of nuclear syndecan-1 enhances histone acetyltransferase (HAT) activity to promote expression of genes that drive an aggressive tumor phenotype. J Biol Chem 2011;286: 30377-83.

32. Masola V, Secchi MF, Gambaro G, Onisto M. Heparanase as a target in cancer therapy. Curr Cancer Drug Targets 2014;14: 286-93.

33. Giordano RJ. Heparanase-2 and syndecan1 in colon cancer: the ugly ducklings or the beautiful swans? Eur J Gastroenterol Hepatol 2008;20:716-8.

34. Yang Y, Macleod V, Miao HQ, Theus A, Zhan F, Shaughnessy JD Jr, et al. Heparanase enhances syndecan-1 shedding: a novel mechanism for stimulation of tumor growth and metastasis. J Biol Chem 2007;282:13326-33.

35. van Wijk XM, van Kuppevelt TH. Heparan sulfate in angiogenesis: a target for therapy. Angiogenesis 2014;17:443-62.

36. Palaiologou M, Delladetsima I, Tiniakos D. CD138 (syndecan-1) expression in health and disease. Histol Histopathol 2014;29: 177-89. 